\title{
Accelerating Parallel Transmit Array B1 Mapping in High Field MRI With Slice Undersampling and Interpolation by Kriging
}

\author{
Guillaume Ferrand*, Michel Luong, Martijn A. Cloos, Alexis Amadon, and Hans Wackernagel
}

\begin{abstract}
Transmit arrays have been developed to mitigate the RF field inhomogeneity commonly observed in high field magnetic resonance imaging (MRI), typically above 3T. To this end, the knowledge of the RF complex-valued $B_{1}$ transmit-sensitivities of each independent radiating element has become essential. This paper details a method to speed up a currently available $B_{1}$-calibration method. The principle relies on slice undersampling, slice and channel interleaving and kriging, an interpolation method developed in geostatistics and applicable in many domains. It has been demonstrated that, under certain conditions, kriging gives the best estimator of a field in a region of interest. The resulting accelerated sequence allows mapping a complete set of eight volumetric field maps of the human head in about $1 \mathrm{~min}$. For validation, the accuracy of kriging is first evaluated against a well-known interpolation technique based on Fourier transform as well as to a $B_{1}$-maps interpolation method presented in the literature. This analysis is carried out on simulated and decimated experimental $B_{1}$ maps. Finally, the accelerated sequence is compared to the standard sequence on a phantom and a volunteer. The new sequence provides $B_{1}$ maps three times faster with a loss of accuracy limited potentially to about $5 \%$.
\end{abstract}

Index Terms-Brain, magnetic resonance imaging (MRI), probabilistic and statistical methods, quantification and estimation.

\section{INTRODUCTION}

$\mathbf{T}$ HE RADIO-FREQUENCY (RF) field nonuniformity in magnetic resonance imaging (MRI) is a well-known source of artefacts in high field MRI [1]. At high frequency (typically above $128 \mathrm{MHz}$, for example in proton MRI, above 3T),

Manuscript received February 27, 2014; revised April 20, 2014; accepted April 27, 2014. Date of publication May 07, 2014; date of current version July 30,2014 . This work was supported by the ISEULT-INUMAC project and the Commissariat à l'Energie Atomique et aux Energies Alternatives (CEA) Asterisk indicates corresponding author.

${ }^{*} \mathrm{G}$. Ferrand is with the Commissariat à l'Energie Atomique et aux Energies Alternatives de Saclay (CEA-Saclay), DSM/IRFU/SACM, F-91191 Gifsur-Yvette, France (e-mail: guillaume.ferrand@cea.fr).

M. Luong is with the CEA-Saclay, DSM/IRFU/SACM, F-91191 Gif-surYvette, France.

M. A. Cloos was with the was with the CEA-Saclay, DSV/I2BM/Neurospin, F-91191 Gif-sur-Yvette, France. He is now with the Bernard and Irene Schwartz Center for Biomedical Imaging, Department of Radiology, New York University School of Medicine, New York, NY 10016 USA.

A. Amadon is with the CEA-Saclay, DSV/I2BM/Neurospin, F-91191 Gifsur-Yvette, France.

H. Wackernagel is with Mines ParisTech, Centre de Géosciences, F-77300 Fontainebleau, France.

Color versions of one or more of the figures in this paper are available online at http://ieeexplore.ieee.org.

Digital Object Identifier 10.1109/TMI.2014.2322440 the RF wavelength in the human head tissues becomes smaller than the sample, leading to a nonuniform transmit-sensitivity profile $\left(B_{1}\right)$ [2], [3]. Specific sequences have been developed to counteract this nonuniformity [4], [5], [6]. These sequences generally lead to long RF pulses or high specific absorption rate in the tissues [7]. During the last decade, transmit arrays have been proposed in order to mitigate these adverse effects [8]. The key principle is to adjust the phase and the amplitude of each of the coil elements. Over the years, many schemes have been developed to optimize the transmit-sensitivity profile [9] (static shimming) or the flip-angle distribution [10]-[13] (dynamic shimming also referred to as transmit-sense).

Each of the above-mentioned solutions requires knowledge of the complex-valued $B_{1}$ field produced by each of the coil elements in the transmit-array. To this end, a dedicated mapping sequence can be used experimentally to map the fields. Over the years, different methods have been proposed to measure the RF field maps. Apart from frequency-modulated pulses technique [14], [15] or the Bloch-Siegert shift technique [16], most $B_{1}$-mapping methods rely on the measurement of the magnetization flip angle as an estimate of the $B_{1}$ magnitude, assuming on-resonance RF excitation. Among these, 3-D steady-state sequences like the Actual Flip angle Imaging sequence [17] allow fairly precise $B_{1}$-profile measurements. However, the minimum duration of these sequences is limited by the specific absorption rate (SAR) and the signal-to-noise ratio (SNR). On the other side, 2-D multislice strategies offer a better SNR to acquisition time ratio, only limited by the longitudinal relaxation time of the tissues, $T_{1}[18]$.

In this latter category, one sequence is particularly suited for the fast calibration of a transmit-array [19] used to control $B_{1}$ profiles via parallel transmission at ultra-high-field. It consists in encoding the flip angle with a preparation saturation pulse immediately preceding an EPI readout [20] or a 2-D-FLASH readout [21]. The ratio between the prepared image and the reference image (without preparation pulse) gives the cosine of the flip angle. A minimal repetition time, $T R$, needs to be allowed so that full $T_{1}$ relaxation occurs before preparing the longitudinal magnetization for the next channel (typically $T R \simeq 5 \cdot T_{1}$, where $T_{1}$ is the longest longitudinal relaxation time known for the sample under study). This is necessary as no steady state other than the thermal equilibrium can be reached throughout the sequence since the preparation pulses induce different excitation patterns at each step. Acceleration methods like EPI, parallel imaging, or even simultaneous multi-slice acquisitions [22] can be used to reduce the acquisition time of a single volume, but 
they cannot fill the dead time induced by the $5-T_{1}$ waiting between the transmit-array channel measurements. This limitation is specific to phased array coils. Adiabatic RESET pulses for defined pre-conditioning of the longitudinal magnetization would allow shortening $T R$, but require high RF power, making the sequence incompatible in vivo because of excess SAR [23]. These sequences applied to the complex $B_{1}$-mapping of an 8-channel transmit-array on a human brain takes about 4 min [21].

Ideally, the calibration of such a transmit array system should be instantaneous as it contains no clinical value. The goal of the present work is to shorten this calibration time to about 1 min. As the repetition time cannot be shortened, we propose to interleave slice and channel-dependent acquisitions while reducing the number of acquired slices. In the case of a 5-mm resolution $B_{1}$ mapping of a human brain, a 4-fold undersampling brings the number of acquired slices from 32 to 8 . The missing slices are then estimated from the undersampled dataset based on an interpolating method well-known in geostatistics, as the $B_{1}$-field varies slowly in space [24].

The idea of interpolating very poorly resolved maps has been studied in the article of Sbrizzi [24]. He proposed to reconstruct poor resolved maps through projection into the space of spherical functions and validates the concept with in vivo field maps. We propose here a different algorithm, the kriging ${ }^{1}$ algorithm, well known in mineral exploration, to reconstruct low resolution maps. The kriging approach was first introduced in the early sixties with Matheron's work on geostatistics [25]. As a branch of statistics, geostatistics focuses on multi-dimensional stochastic processes. It aims at deriving the maximum information from noisy and low resolution maps. Except the condition that the quantity to be estimated varies slowly in space, no other physical assumption is required. The kriging algorithm allows estimation of an expected value, based on a statistical quantity commonly referred to as a variogram [26]. This algorithm has already been applied to other biomedical issues [27] as well as to electromagnetic problems [28]. It is essential to remember the impossibility of demonstrating that kriging is applicable to a specific problem a priori. As usual in statistics, assumptions must be made first, which validity in only checked a posteriori with an appropriate test.

The potential of the kriging method is explored in the context of in vivo $B_{1}$ calibration of an 8-channel transmit array at $7 \mathrm{~T}$. Results are compared to a sinc interpolation and to the method developed by Sbrizzi [24].

\section{MATERIALS AND MethodS}

\section{A. Elements of Kriging}

The kriging method was first developed for mineral and oil prospection in the $1960 \mathrm{~s}$. It aimed at locally estimating the potential wealth of a mine or an oil field with a series of drillings, in order to optimize the exploitation of the resource. For this purpose, engineers considered the local wealth of the mine, at

\footnotetext{
${ }^{1}$ The term "krige" comes from the name of the geologist Danie Gerhardus Krige, who was the first mining engineer to apply geostatistics to evaluate gold resources in South Africa.
}

position $\vec{r}$, as a random variable, $Z(\vec{r})$. Each drilling was considered as a measurement, a realization in a statistical point of view, of $Z$ at position $\vec{r}_{0}: Z_{m}\left(\vec{r}_{0}\right)$.

The question was: what can we expect to find at position $\vec{r}$, knowing what we found at different positions $\left\{\vec{r}_{1}, \ldots, \vec{r}_{n}\right\}$ ? Kriging was the answer proposed by Matheron [25]. The method is based on the concept of variogram. If we assume that the expected value, in terms of statistics, of the square of the difference $Z(\vec{r}+\vec{h})-Z(\vec{r})$, does not depend on $\vec{r}$, which is usually called the stationarity assumption, we can define the variogram $\gamma$ by

$$
\gamma(\vec{h})=\frac{1}{2} E\left((Z(\vec{r}+\vec{h})-Z(\vec{r}))^{2}\right)
$$

where $E$ denotes the expected value.

The variogram can be estimated for every $\vec{h}$ with a small number of measurements. Variogram models generally involve two or three parameters estimated based on these measurements. Furthermore, Matheron showed that the kriging estimator $Z_{K}(\vec{r})$ is the best linear unbiased estimator knowing a series of realizations of $Z$ at positions $\left\{\vec{r}_{1}, \ldots, r_{n}\right\}$

$$
Z_{K}(\vec{r})=\sum_{a=1}^{n} \omega_{a} Z_{m}\left(\vec{r}_{a}\right)
$$

where $\left\{\omega_{1}, \ldots, \omega_{n}\right\}$ is the solution of the linear equation system

$$
\left\{\begin{array}{c}
\text { for all } b \in\{1, \ldots, n\} \\
\sum_{a=1}^{n} \omega_{a} \gamma\left(\vec{r}_{b}-\vec{r}_{a}\right)+\mu=\gamma\left(\vec{r}_{b}-\vec{r}\right) \\
\sum_{a=1}^{n} \omega_{a}=1 .
\end{array}\right.
$$

This linear system has $n+1$ equations and $n+1$ unknowns; it always has a unique solution. The additional parameter $\mu$ is a Lagrange multiplier used in the minimization of the kriging error to satisfy the unbiasedness condition. It has no physical meaning. The theoretical foundation of kriging is detailed by Wackernagel [29]. Its presentation is far outside of the scope of this article. The model and fitting algorithm chosen for this study will be described below.

Kriging was first used with real positive quantities such as mass concentration of minerals, oil, etc. Most of the algorithms were developed for 2-D or 3-D experiments with real values. However, by simply splitting complex-valued numbers into real and imaginary parts, these kriging algorithms can be easily applied to each part. The kriging algorithm is effective only if there is a spatial correlation in the field being explored. In other words, the variogram must not be a constant function for $\vec{h} \neq 0$. For electromagnetic field mapping, the efficiency of the kriging approach has already been demonstrated in different experiments [28].

\section{B. Kriging Interpolation Algorithm}

We propose to apply the kriging algorithm to field maps of resolution $48 \times 48 \times 8$, respectively in directions $x, y$ and $z$, in order to produce $48 \times 48 \times 32$ field maps. The $z$ axis refers hereinafter to the slice-encoding direction, which corresponds to the $B_{0}$ field direction. The principle of the kriging algorithm can be 
easily applied to other resolutions. The voxel size of the interpolated field maps is defined to $5 \times 5 \times 5 \mathrm{~mm}^{3}$, such as the field of view is $240 \times 240 \times 160 \mathrm{~mm}^{3}$. In the following paragraphs, $\delta l$ defines the voxel size equal to $5 \mathrm{~mm}$. Consequently, the presented algorithm aims at multiplying the resolution by a factor 4 along $z$-axis. A triplet of natural numbers $\left(i_{a}, j_{a}, k_{a}\right)$ defines the position $\vec{r}_{a}=\left(x_{a}, y_{a}, z_{a}\right)$ in the Cartesian grid whose origin is located at a corner of the volume of interest, such as $x_{a}=i_{a} \cdot \delta l$, $y_{a}=j_{a} \cdot \delta l$, and $z_{a}=k_{a} \cdot \delta l$. The initial dataset, that is the $48 \times 48 \times 8$ initial field map, is embedded in two 3-D arrays of size $48 \times 48 \times 32$ called $B_{R}$ and $B_{I}$ corresponding respectively to the real and imaginary part. Each of these two 3-D arrays will define successively the domain of kriging. Their construction is obtained element by element as follows:

$-B_{R}\left(i_{a}, j_{a}, k_{a}\right)=N a N$ and $B_{I}\left(i_{a}, j_{a}, k_{a}\right)=N a n$ ("Not-a-Number") if $\vec{r}_{a}$ is located on an unmeasured slice or outside of the sample.

- Otherwise, $B_{R}\left(i_{a}, j_{a}, k_{a}\right)=\operatorname{Re}\left(B_{1}\left(\vec{r}_{a}\right)\right)$ and $B_{I}\left(i_{a}, j_{a}, k_{a}\right)=\operatorname{Im}\left(B_{1}\left(\vec{r}_{a}\right)\right)$.

At least three-quarters of $B_{R}$ and $B_{I}$ is filled with $N a n$ values at the end of this process.

First, we must define the set of positions $\left\{\vec{r}_{1}, \ldots, \vec{r}_{n}\right\}$ used to estimate $E\left(B_{R}(\vec{r})\right)$ or $E\left(B_{I}(\vec{r})\right)$ in (1). Geostatistics proves that, under the stationarity hypothesis, the correlation between two measurements decreases, or stay constant, when the distance increases: there is statistically less information between two distant locations compared to neighboring locations. Consequently, it is not necessary to include all the measured values of $B_{R}$ or $B_{I}$ to estimate $E\left(B_{R}(\vec{r})\right)$ or $E\left(B_{I}(\vec{r})\right)$. Moreover, the higher the number of positions $\mathrm{n}$, the higher the computational time in kriging. Another criterion for choosing the set the positions $\left\{\vec{r}_{1}, \ldots, \vec{r}_{n}\right\}$ states that all measurements cannot be located on the same slice. Otherwise, the variation along z-axis would be ignored by the algorithm. In our study, the compromise consists in defining $\left\{\vec{r}_{1}, \ldots, \vec{r}_{n}\right\}$ such as all the measured values are included in a 25 -mm-radius sphere centered at $\vec{r}$. The value of $25 \mathrm{~mm}$ is higher than the distance between two measured slices $(20 \mathrm{~mm})$, ensuring that measurements are taken on two adjacent slices. In the same time, this sampling rule excludes all the measurements that would not improve significantly the kriging. The maximum distance between two positions in the $25-\mathrm{mm}$-radius sphere is $50 \mathrm{~mm}$. Hence, the eligible values for the variable $h$ in the variogram function can be limited in a range of $50 \mathrm{~mm}$.

Among the numerous possible variogram models proposed in [29], we chose a versatile and widely used model which is entirely defined by only three parameters

$\gamma\left(\vec{h}, p_{1}, p_{2}, p_{3}\right)$

$= \begin{cases}0, & \text { if } h=0 \\ p_{1}+p_{2}\left(\frac{h}{p_{3}}\right)^{2} & \\ \quad \times\left[7-\frac{h}{p_{3}}\left[\frac{35}{4}-\left(\frac{h}{p_{3}}\right)^{2}\left[\frac{7}{2}-\frac{3}{4}\left(\frac{h}{p_{3}}\right)^{2}\right]\right]\right], & \text { if } 0<h<p_{3} \\ p_{1}+p_{2}, & \text { otherwise }\end{cases}$

As an isotropic model, this variogram does not depend on the direction of $\vec{h}$. It is able to fit various experimental data with a minimum number of parameters: $p_{1}, p_{2}$ and $p_{3}$. The variance of the random variable $Z$ is given by $p_{1}+p_{2}$. The minimum correlation between two adjacent positions is represented by $p_{1}$. Finally, $p_{3}$ is the range of the model that corresponds to the distance $h$ from which the variogram becomes constant.

The structure of the field dataset, a set of discrete values on a Cartesian grid, allows only discrete values for $h$, the distance between two positions $\vec{r}_{a}$ and $\vec{r}_{b}$ on the grid. Each value must be written as the square root of a sum of perfect squares multiplied by $\delta l$, namely $h=\delta l \sqrt{i^{2}+j^{2}+k_{2}}$.

In order to estimate the real-part variogram $\gamma_{R}$ parameters $\left(p_{1}, p_{2}\right.$ and $\left.p_{3}\right)$, we proceed as follows.

- For every eligible discrete value $h$ between 0 and $50 \mathrm{~mm}$, we initialize two functions, $W(h)=0$ and $\Gamma(h)=0$.

- For every couple $\left(\vec{r}_{a}, \vec{r}_{b}\right.$ in the domain of kriging:

- If $\left(B_{R}\left(i_{a}, j_{a}, k_{a}\right) \neq N a N\right)$ and $\left(B_{R}\left(i_{b}, j_{b}, k_{b}\right) \neq\right.$ Nan) and $\left(\left\|\vec{r}_{a}-\vec{r}_{b}\right\| \leq 50 \mathrm{~mm}\right)$.

- Define $h=\left\|\vec{r}_{a}-\vec{r}_{b}\right\|$.

- Add 1 to $W(h)$.

- $\operatorname{Add}(1 / 2)\left(B_{R}\left(i_{a}, j_{a}, k_{a}\right)-B_{R}\left(i_{b}, j_{b}, k_{b}\right)\right)^{2}$ to $\Gamma(h)$.

- Define the experimental variogram by: $G(h)=$ $\Gamma(h) / W(h)$.

— Find $\left(p_{1}, p_{2}, p_{3}\right)$ that minimizes $J$ with

$$
J=\sum_{h} W(h)\left[G(h)-\gamma_{R}\left(h, p_{1}, p_{2}, p_{3}\right)\right]^{2} .
$$

The fitting process consists in a weighted least square minimization between the experimental variogram $G$ and the chosen variogram model, where the weight $W$ represents the number of measurements in the whole domain of kriging, corresponding to a value of $h$. The imaginary part variogram, $\gamma_{I}$ is estimated with the same algorithm applied to $B_{I}$. The ability of fitting the variogram model is comparable by analogy to the chi-squared test in statistics: it checks the validity of the stationarity hypothesis.

Once both variogram models are defined for real and imaginary parts, the kriging itself can start. The algorithm for kriging $B_{R}$ is the following:

For every position $\vec{r}$, or equivalently every triplet $(i, j, k)$, in the domain of kriging.

- If $\left(B_{R}(i, j, k)=N a n\right)$ and ( $\vec{r}$ corresponds to a position inside of the sample).

- Identify the set $\left\{\vec{r}_{1}, \ldots, r_{n}\right\}$ or $\mathrm{n}$ triplets corresponding to all the measured values located in a 25 -mm-radius sphere centered at $\vec{r}$.

- Build and solve the system of linear equations according to (3) with $\gamma=\gamma_{R}$.

- Compute $B_{R}(i, j, k)$ according to (2): $B_{R}(i, j, k)=$ $\sum_{a=1}^{n} \omega_{a} B_{R}\left(i_{a}, j_{a}, k_{a}\right)$.

The same algorithm is then applied to $B_{I}$ to give $B_{K}=$ $B_{R}+i B_{I}$ finally. At the top and bottom of the brain, the set $\left\{\vec{r}_{1}, \ldots, r_{n}\right\}$ can be entirely included in one instead of two slices, reducing locally the accuracy of the algorithm. In the following paragraph, another interpolation method is proposed for comparison.

\section{Zero-Padding sinc Interpolation Algorithm}

In signal processing, one well-known interpolation technique is the sinc interpolation with zero-padding algorithm [30], [31]. 
It consists in Fourier transforming the time signal, adding zero values on the edges of the spectrum and finally reverse Fourier transforming back in the time domain. This technique tends to produce Gibbs ringing effects [30], which could not mitigated efficiently by apodization, for instance with Hann window, due to the limited number of samples. However, sinc interpolation is deemed to provide a fast algorithm and can operate directly on complex quantities. Hence, it appears as a good benchmark. The benchmark is implemented to increase four-fold the space resolution in one direction. Let define $B_{1}^{M}=B_{R}+i B_{I}$ the complex-valued 3-D array of size $48 \times 48 \times 32$ from the real part and imaginary part arrays used in Section II-B. The indices and triplets references are the same. Operating line by line, the interpolation algorithm implementation is very similar to time signal interpolation.

For every couple $(i, j)$ or equivalently for every line along $z$-axis in $B_{1}$ array.

- Define a vector $B_{v}$ with 32 elements such as $B_{v}(k)=$ $B_{1}^{M}(i, j, k)$.

- Define a vector $B_{v u}$ of 8 elements from $B_{v}$ containing field values on the measured slices only.

- Replace $N a n$ (voxels outside the sample) by zero in $B_{v u}$ and Fourier transform $B_{v u}$ into $F B_{v u}$.

- Pad $F B_{v u}$ with zero values to get a vector of the same size as $B_{v}$ and reverse Fourier transform $F B_{v u}$ into $B_{\text {vint }}$.

- Use $B_{\text {vint }}$ to construct line by line the interpolated $B_{1 \text { int }}$ array.

Due to the fact that only eight measured values are involved in the zero-padding process, the images corresponding to $B_{1 i n t}$ appear very noisy and affected by Gibbs effects. They would not be usable without an additional smoothing. This last step is realized by the convolution with a $3 \times 3 \times 3$ square-box filter applied to $B_{1 i n t}$. The result is called $B_{F}$.

\section{Standard and Undersampled XFL Sequences}

Now that the kriging interpolation algorithm and its benchmark are presented, it is necessary to consider an experimental $B_{1}$-mapping technique. A technique using a magnetization preparation RF pulse with TurboFLASH readout was first described for single-channel RF coils [18]. It consists in encoding the flip angle with a preparation saturation pulse immediately preceding a centric-ordered 2-D FLASH readout. Its version for multiple transmission RF coils will be referred as multiple transmission multislice $B_{1}$-mapping technique with FLash readout, or XFL sequence to make it shorter. For the implementation [21], the preparation pulse is made very selective and its spatial profile encompasses that of the imaging pulse. Moreover, for the calibration of an $N$-channel transmit-array, whereas the prepared flip angle depends on the transmit-channel under scrutiny, the imaging pulse of the 2-D FLASH readout is played out on all channels in the same combination (the circularly polarized mode, or CP-mode) as that used for the reference image. The sequence is repeated for all $S$ slices and all $N$ channels with the following order of acquisition.

1) Reference images: slice $1,3,5, \ldots, S-1$, then slice $2,4, \ldots$, S. (This interleaved fashion prevents cross-talk between adjacent slices.)
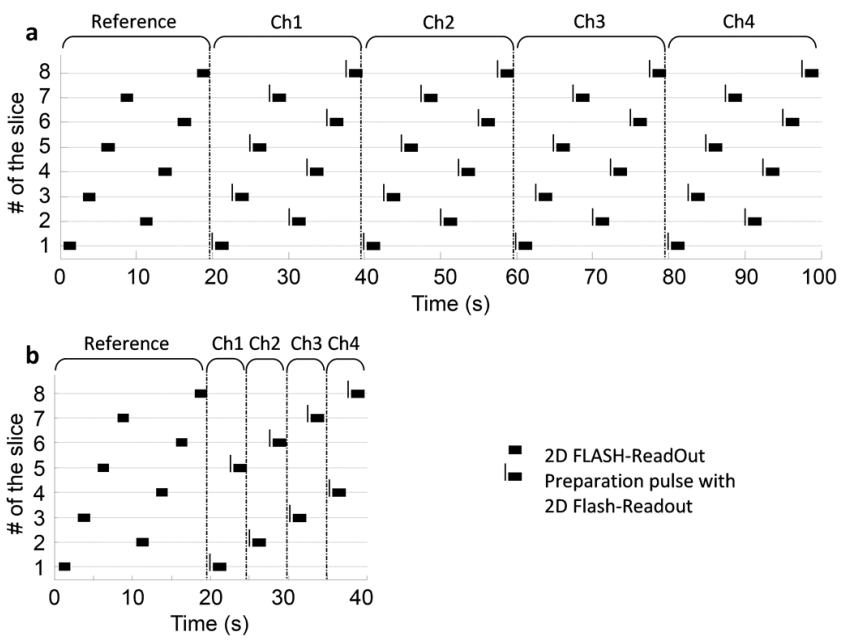

Fig. 1. Standard XFL sequence for eight slices and four channels (a) and SU-XFL sequence with slices and channels interleaving (b).

2) Channel 1-prepared images: slice $1,3,5, \ldots, S-1$, then slice $2,4, \ldots, S$.

3) Channel 2-prepared images: slice $1,3,5, \ldots, S-1$, then slice $2,4, \ldots, S$.

4) $\ldots N+1$. Channel $N$-prepared images; slice $1,3,5, \ldots, S-1$, then slice $2,4, \ldots, S$.

An example is shown on Fig. 1(a) for a standard XFL sequence with $N=4$ and $S=8$. The reference image ("Reference") is acquired without preparation pulse. The flip angle, and hence the $B_{1}$ field amplitude, is obtained by dividing prepared-images by reference images; details are given in [21]. Moreover, in addition to the $B_{1}$ amplitude maps, transmit-array calibration requires the relative $B_{1}$ phase maps. These phase maps can be acquired in $N$ additional steps whereby no preparation pulse is played [21]. The phase of the field maps is defined as the relative phase to the reference image. The acquisition time of the phase field maps is not limited by $T_{1}$, only by SAR and SNR.

The slice undersampled XFL sequence, hereafter called SU-XFL sequence uses the same scheme than the standard XFL sequence except that we take advantage of the time not used to acquire more slices to interleave acquisition on other channels. The acquisition of reference images does not change. For an undersampling factor of 4 , channel 1 is only acquired for slices $1,5 \ldots, S-3$, channel 2 for slices $2,6 \ldots, S-2$ and so on until channel 4 . If there are more than four channels, the same sequencing applies to the group of channel 5 to channel 8: channel 5 is only acquired for slices $1,5 \ldots, S-3$, channel 6 for slices $2,6 \ldots, S-2$, etc. An illustration is given on Fig. 1(b). The same interleaving scheme is used for the phase maps (not shown in Fig. 1). As with the standard XFL sequence, the phase of the field maps is defined as the relative phase to the reference image. This relative phase is well defined for all the slices because the reference image is acquired without undersampling. Moreover, the reference image is the same for XFL and SU-XFL, which ensures the consistency of the phase between both acquisitions.

In this study, it should be recalled that the standard XFL sequence will produce a field map of resolution $48 \times 48 \times 32$ for 
every channel while the SU-XFL sequence will produce a field map of resolution $48 \times 48 \times 8$ before interpolation.

\section{E. Interferometry for $B_{1}$-Mapping}

In Section II-D, the term "channel" may refer to one physical channel or to a mode resulting from a combination of several physical channels. Practically, experimental $B_{1}$-mapping rarely acquires individual physical channel one after another because the SNR is not optimal [32]: an interferometry approach is preferred generally [33]. This is also the choice made in this work.

Let $B_{k}^{c h}$ denote the field map, $B_{1}$, produced by physical channel $k$ for $1 \mathrm{~V}$ at the input. A set of phases $\theta_{k}$ was previously worked out, in such a way that, sending the same power on every input of the coil with phase $\theta_{k}$ for channel $k$, gives a field pattern close to the well-known circularly polarized (CP) mode of a birdcage coil [34]. This pseudo CP-mode, $B^{C P}$ should not show a location with zero-field (field level below the noise level) in the brain. This condition was verified experimentally with many volunteers. For a given voltage amplitude $\mathrm{v}$ at every input, $B^{C P}$ can be written

$$
B^{C P}=v \sum_{k=1}^{N} B_{k}^{c h} e^{i \theta_{k}} .
$$

The interferometry method consists in acquiring $B_{k}$ defined by

$$
B_{k}=B^{C P}-2 v B_{k}^{c h} e^{i \theta_{k}} .
$$

With the knowledge of $\theta_{k}$ and $B_{k}$ for every $k$, solving the system of linear equations provided by (6) and (7) returns $B_{k}^{c h}$ for every channel. The computation of $B_{k}^{c h}$ is always the last step of the process, after interpolation of the undersampled data, because the measured data are slice-interleaved in the SU-XFL sequence. Hence, the error of interpolations will be evaluated on $B_{k}$ and $B_{k}^{c h}$, before and after channel-by-channel reconstruction.

\section{F. Experimental Setup and Method of Validation}

The demonstration of the method makes use of an eight-independent-channel transceive array (head coil) designed for a 7T Siemens Magnetom scanner equipped with the multiple-transmit array capability. This coil consists of eight dipoles for transmission and reception. The resonance frequency of the dipoles is adjusted to the Larmor frequency of the proton at 7T. The dipoles were previously described in [35]. The power budget at the input of each channel is limited to 650 $\mathrm{W}$. More details about the operation of this transmit-array for in vivo experiments are found in [34]. The validation of interpolation algorithms and SU-XFL sequence follows different steps.

In a first step, the kriging algorithm and its benchmark are tested on simulated field maps for a human brain in an attempt to qualify the interpolation algorithms accuracy regardless the experimental $B_{1}$-mapping sequences and their potential intrinsic limitations. The simulated maps are obtained by full-wave simulations with the HFSS code based on the finite element method. The anatomical model and the details for simulations have already been described in a previous work [36].
An initial set of eight simulated maps, one for each dipole, is produced with a resolution $48 \times 48 \times 32$. By decimation, another set of resolution $48 \times 48 \times 8$ is also produced to mimic the slice undersampling. They are interpolated using kriging and zero-padding algorithms to be compared to the initial dataset. For the comparison based on simulation, $B_{\text {ref }}$ corresponds to a simulated map of $B_{1}$ in the initial set. It has the structure of $B_{1}$ as defined in Section II-C. The error due to the interpolation is denoted by $e_{p}^{\mathrm{int}}=\left\|B_{p}-B_{\text {ref }}\right\| /\left\|B_{\text {ref }}\right\|$ with $p=\{K, F\}$, where $K$ and $F$ refer respectively to interpolation with kriging and zero-padding. The norm $\|\cdot\|$ applied to the 3 -D array $B$ is defined by $\|B\|=\sqrt{\sum_{i} \sum_{j} \sum_{k}|B(i, j, k)|^{2}}$.

In a second step, a set of $B_{1}$-field maps of resolution $48 \times$ $48 \times 32$ is acquired with the interferometry method on a volunteer with the standard XFL sequence. These field maps are then decimated on a computer, according to the scheme of the SU-XFL sequence, to obtain $48 \times 48 \times 8$ field maps. The results of interpolation with kriging and zero-padding algorithms are compared to the initial field map for both $B_{k}$ and $B_{k}^{c h}$ maps. This step requires checking that the movements of the volunteer are limited during the sequence acquisition. This control is performed by an alignment test on the images. Under these conditions, this step allows estimating the accuracy of both interpolation algorithms with a single dataset. At this stage, we can compare our results with the ones obtained by Sbrizzi et al. [24]. In this article, the authors proposed to interpolate in vivo field maps for slices $20 \mathrm{~mm}$ away with a very different method based on spherical functions decomposition. They also operated at $7 \mathrm{~T}$ and adopted the same metric for the error evaluation.

In a third step, it is essential to qualify the stability of the standard XFL sequence before any comparison to the SU-XFL sequence. A 21 saline-agar spherical phantom (dielectric constant $=72$, conductivity $=0.9 \mathrm{~S} / \mathrm{m}$ ) is used in that purpose: it avoids artefacts attributable to the movements of a volunteer. Two consecutive acquisitions with identical parameters provide an indication on the minimum error that cannot be reduced while comparing SU-XFL sequence against standard XFL sequence. This error is denoted $\Delta=\left\|B_{1 a}-B_{1 b}\right\| /\left\|B_{1 a}\right\|$, where $B_{1 a}$ and $B_{1 b}$ represent 3 -D arrays of dimension $48 \times 48 \times 32$ containing respectively measured complex-valued $B_{1}$ field in the first and the second acquisition.

In a fourth and last step, the interpolated field maps acquired using the SU-XFL sequence are compared to the ones obtained by the standard XFL sequence for a phantom on the one hand and on a volunteer on the other. Each sequence is run successively. For in vivo mapping, there is a risk that the volunteer may have moved slightly his head in between the two sequences. Therefore, precautions must be taken while analyzing the experimental results in this step.

\section{RESULTS}

\section{A. Interpolation of Simulated Field Maps}

The interpolation by kriging of eight simulated field maps gives an error $e_{K}^{\text {int }}$ between $3.5 \%$ and $5.8 \%$ from one channel to another, with an average value of $4.5 \%$. At the same time, the sinc interpolation gives an error $e_{F}^{\text {int }}$ between $19.3 \%$ and 
TABLE I

Interpolation ERrors on IN VIVo Field Maps. Labels “Interf." and “Channel” Refer Respectively to Measured InTERferences and Channels After ReCONSTRUCTION ACCORDING to (6) AND (7). NUMBERS 1 to 8 IN THE HEADER REFER EITHER TO THE NUMBER OF THE INTERFERENCE OR TO THE Number of the Channel. Rows (I) to (IV) Relate to Results Obtained With a Decimated Dataset Acquired By the Standard XFL SEQuence. Rows (V) to (VIII) Relate to Results ObTaINED In Comparing SU-XFL SEQUENCE to the STANDARd XFL SEQUENCE

\begin{tabular}{cccccccccc}
\hline \hline Decimated & 1 & 2 & 3 & 4 & 5 & 6 & 7 & 8 & Mean \\
\hline (i) $e_{F}^{i n t}$-interf. & $19.2 \%$ & $20.6 \%$ & $21.5 \%$ & $21.6 \%$ & $22.0 \%$ & $21.4 \%$ & $21.3 \%$ & $19.6 \%$ & $20.9 \%$ \\
(ii) $e_{K}^{\text {int }}-$ interf. & $2.4 \%$ & $2.8 \%$ & $2.9 \%$ & $5.0 \%$ & $3.0 \%$ & $3.5 \%$ & $3.0 \%$ & $4.2 \%$ & $3.4 \%$ \\
(iii) $e_{F}^{i n t}$-channel & $25.2 \%$ & $23.9 \%$ & $22.7 \%$ & $27.0 \%$ & $21.0 \%$ & $21.2 \%$ & $22.0 \%$ & $26.7 \%$ & $23.7 \%$ \\
(iv) $e_{K}^{i n t}-$ channel & $6.9 \%$ & $5.5 \%$ & $3.8 \%$ & $5.2 \%$ & $3.8 \%$ & $4.6 \%$ & $5.7 \%$ & $6.7 \%$ & $5.3 \%$ \\
\hline \hline SU-XFL versus XFL & 1 & 2 & 3 & 4 & 5 & 6 & 7 & 8 & Mean \\
\hline (v) $e_{F}^{i n t}-$ interf. & $25.2 \%$ & $27.7 \%$ & $28.5 \%$ & $25.9 \%$ & $30.2 \%$ & $29.9 \%$ & $28.4 \%$ & $22.0 \%$ & $27.2 \%$ \\
(vi) $e_{K}^{i n t}-$ interf. & $16.4 \%$ & $17.3 \%$ & $20.5 \%$ & $16.8 \%$ & $20.5 \%$ & $18.8 \%$ & $21.3 \%$ & $11.6 \%$ & $17.9 \%$ \\
(vii) $e_{F}^{i n t}-$ channel & $44.5 \%$ & $38.3 \%$ & $34.4 \%$ & $27.6 \%$ & $27.4 \%$ & $33.3 \%$ & $41.1 \%$ & $37.2 \%$ & $35.5 \%$ \\
(viii) $e_{K}^{i n t}-$ channel & $32.8 \%$ & $26.1 \%$ & $24.7 \%$ & $17.1 \%$ & $19.1 \%$ & $23.6 \%$ & $37.5 \%$ & $24.1 \%$ & $25.6 \%$ \\
\hline \hline
\end{tabular}

TABLE II

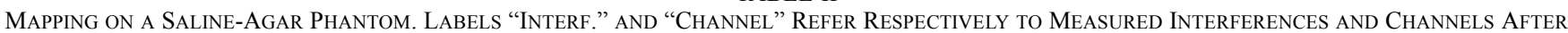

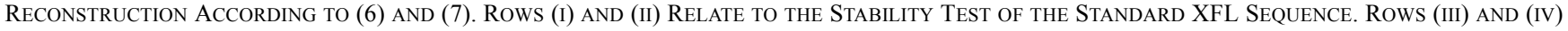
ReLATE to the COMParison OF SU-XFL AND Standard XFL SEQUENCES

\begin{tabular}{cccccccccc}
\hline \hline Stability & 1 & 2 & 3 & 4 & 5 & 6 & 7 & 8 & Mean \\
\hline (i) $\Delta$-interf. & $7.8 \%$ & $7.4 \%$ & $7.7 \%$ & $7.4 \%$ & $7.7 \%$ & $6.5 \%$ & $7.9 \%$ & $7.6 \%$ & $7.5 \%$ \\
(ii) $\Delta$-channel & $10.0 \%$ & $11.1 \%$ & $9.6 \%$ & $8.2 \%$ & $8.3 \%$ & $7.5 \%$ & $7.8 \%$ & $8.2 \%$ & $8.9 \%$ \\
\hline \hline SU-XFL versus XFL & 1 & 2 & 3 & 4 & 5 & 6 & 7 & 8 & Mean \\
\hline (iii) $e_{F}^{\text {int }}$-interf. & $8.7 \%$ & $9.2 \%$ & $9.5 \%$ & $9.8 \%$ & $10.4 \%$ & $9.6 \%$ & $12.4 \%$ & $11.8 \%$ & $10.2 \%$ \\
(iv) $e_{K}^{\text {int }}$-channel & $15.3 \%$ & $15.4 \%$ & $16.1 \%$ & $12.4 \%$ & $12.0 \%$ & $13.6 \%$ & $13.4 \%$ & $13.7 \%$ & $14.0 \%$ \\
\hline \hline
\end{tabular}

$22.5 \%$ with an average value of $21.4 \%$. In the interpolation based on zero-padding, the information used in the process is only taken along the z-axis, even if the smoothing filter provides an averaging over the three directions afterwards. On the contrary, kriging takes information from measurements in all directions systematically in one shot; hence the better accuracy. Zero-padding is much faster: it runs in less than $1 \mathrm{~s}$ to complete the whole process on a workstation (2 CPUs, Intel X5670) while kriging requires about $20 \mathrm{~s}$.

\section{B. Interpolation of Decimated In Vivo Field Maps}

The results (Table I) reported in this section correspond to the second step in the validation method using a decimated in vivo dataset, interpolated with the zero-padding algorithm $\left(e_{F}^{\mathrm{int}}\right)$ or the kriging algorithm $\left(e_{K}^{\mathrm{int}}\right)$. In all cases, the error between interferences and between channels shows a small spread, which indicates that both interpolation techniques are not significantly dependent on the field patterns. The error obtained with kriging is at least six times lower compared to the zero-padding interpolation. Due to the definition of the interpolation error, which is normalized to the norm of the reference field map, this error always appear slightly lower, by less than $40 \%$, for interferences compared to channels. Indeed, the average value of $B_{1}$ over the field of view is always higher for the interference considering a given available total power: this is also the foundation of the interferometry approach for $B_{1}$-mapping.

The interpolation error averaged over the eight interferences or channels may be compared to the error, defined identically, obtained by another interpolation method for a CP-mode. In a recent work [24], an error of $22 \%$ was reported by their authors while attempting to interpolate in vivo field maps of a human brain at $7 \mathrm{~T}$ between slices $20 \mathrm{~mm}$ away. These parameters are identical to the ones of the current study. With the kriging algorithm, the average error only amounts to $3.4 \%$ and $5.3 \%$, respectively, for the interferences and the channels. The interpolation based on kriging appears then at least four times more accurate.

\section{Stability of the Standard XFL Sequence With a Phantom}

The standard XFL sequence has its own limitation in reproducibility mainly because of the complex behavior of the spoiling and other noise sources in the experimental setup. Even if the same sequence is run twice, the measured field maps will be different: they are affected by an error that can be referred to as experimental. This error is evaluated in this third step of validation. Results [Table II rows (i) and (ii)] show values of $7.5 \%$ and $8.9 \%$ depending on whether the $B_{k}$ interference maps or the $B_{k}^{c h}$ channel maps are considered. It is worth noting that this experimental error is higher than the kriging interpolation error analyzed in Section III-B.

\section{Comparison of SU-XFL Sequence to Standard XFL Sequence}

To complete this study, it appears instructive to compare the field maps obtained with both sequences, even if the difference in the results does not depend on the accuracy of the interpolation algorithm only. The global error $e_{K, F}^{\mathrm{int}}$ includes interpolation error, experimental error and also displacement error in the case of a volunteer. In this comparison, the analysis is focused on the reconstructed channel-by-channel $B_{k}^{c h}$ field maps since they are the ones desired in the $B_{1}$-calibration for parallel transmission in MRI. The results for the $B_{k}$ interferences are nevertheless given for information.

The average error for in vivo channel-by-channel maps [Table I row (viii)] amounts to $25.6 \%$ to be compared to $14 \%$ [Table II row (iv)] for the phantom. This difference may be 

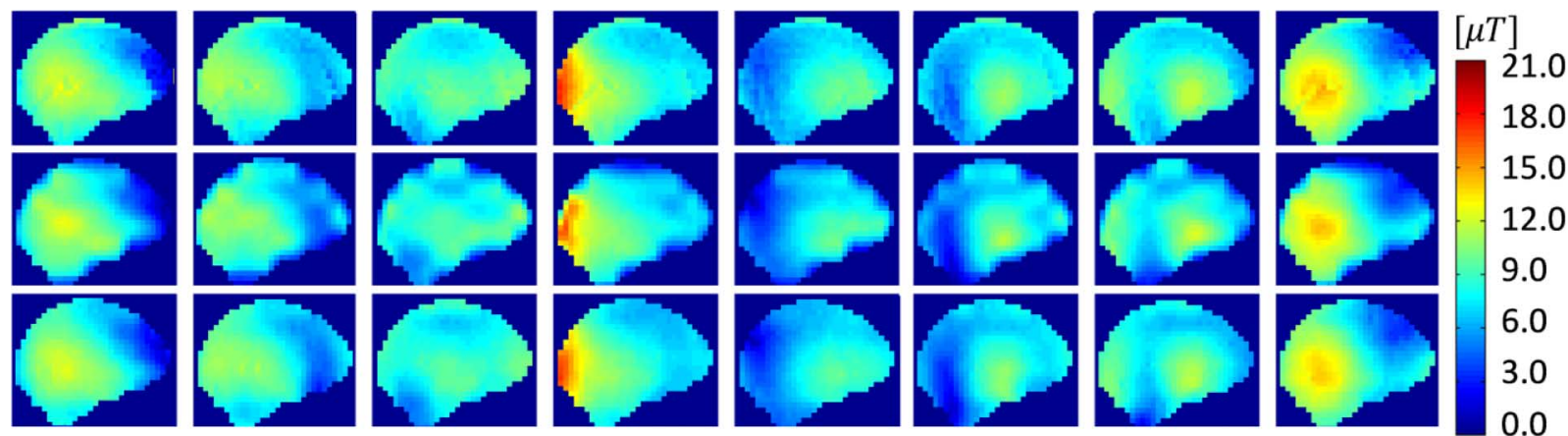

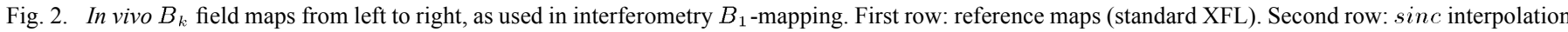
(SU-XFL). Third row: Kriging interpolation (SU-XFL). Central sagittal slices, interpolation along superior-inferior axis.
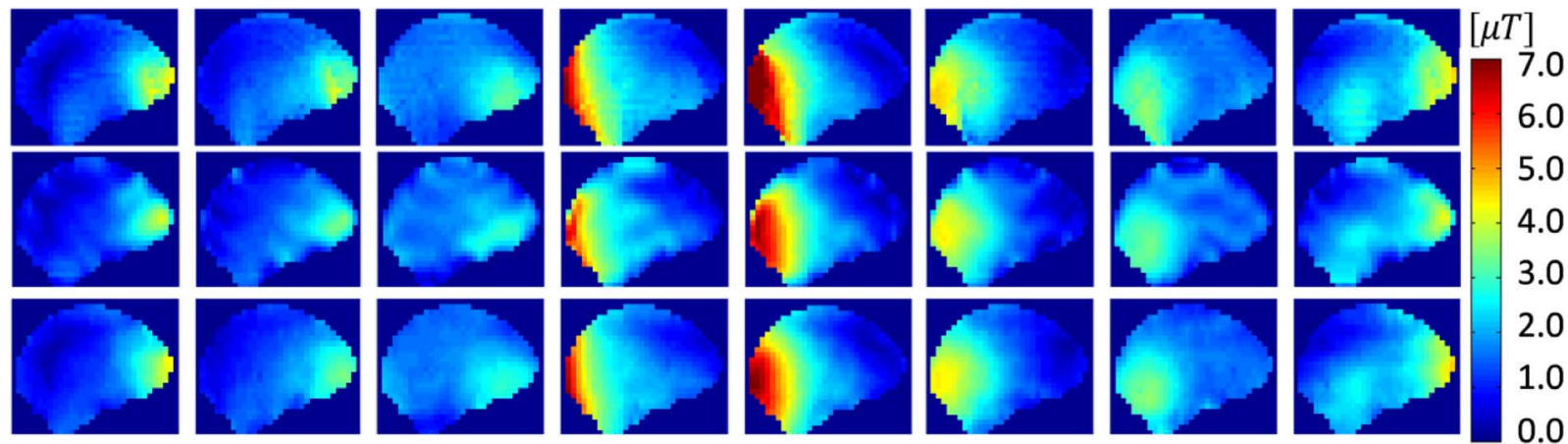

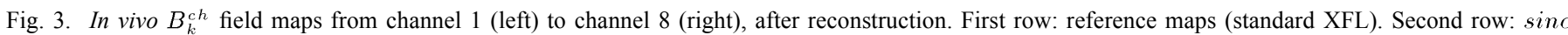
interpolation (SU-XFL). Third row: Kriging interpolation (SU-XFL). Central sagittal slices, interpolation along superior-inferior axis.

explained by the displacement of the head: the standard XFL sequence lasts $4 \mathrm{~min}$ followed by a break about $5 \mathrm{~min}$, the time necessary to configure the scanner for the SU-XFL sequence that lasts $1 \mathrm{~min} 15 \mathrm{~s}$. A detailed analysis of the error spread from one channel to another supports this explanation since the maximum error to minimum error ratio rises from 1.3 for the phantom to 2.2 for the volunteer. Globally, the results obtained with the zero-padding algorithm are always less accurate: the average error for in vivo maps amounts to nearly $40 \%$. In order to provide an overview on how all those error values are translated visually on in vivo field maps, Figs. 2 and 3 display the amplitude of the latter on the central sagittal plane, respectively, for the eight interferences and the eight channels. The images show that the kriging algorithm in the SU-XFL sequence provides faithful maps, even close to the limits of the region of interest, where the number of data is poor in the neighborhood of the kriged locations.

\section{Discussion AND CONCLUSION}

The variation of the $B_{1}$-field is smooth in the human head or chest, even at 7T: it is theoretically possible to interpolate very low-resolution datasets, and thus, to reduce the acquisition time of the calibration. In this article, we proposed a kriging algorithm to interpolate the datasets. The error only due to the interpolation technique was assessed as typically $5 \%$. This was six times lower compared to a zero-padding algorithm. The kriging was also compared with the results reported by Sbrizzi et al. [24] in similar conditions. While it is not surprising that kriging is more accurate than the sinc interpolation, which is known to produce Gibbs artefacts [30], it is worth noting that the kriging interpolation is also much more accurate than interpolation with spherical function decomposition proposed by Sbrizzi. First developed for mining exploration, where each measurement is very money and time demanding, it showed here to be relevant for interpolating low resolution $B_{1}$-maps.

Combining the kriging algorithm to the XFL $B_{1}$-mapping sequence has provided a new mapping sequence we called SU-XFL sequence, which turned out to be at least three times faster. The SU-XFL sequence was able to calibrate $B_{1}$ maps of an 8-channel transmit array in $1 \mathrm{~min}$ and $15 \mathrm{~s}$. The comparison of both sequences on a 21 saline-agar phantom indicated an average error of $14 \%$ which encompassed an experimental error of $9 \%$, assessed with two successive identical acquisitions using the standard XFL sequence. The results on a saline phantom show that the accuracy is not significantly degraded when shortening significantly the $B_{1}$ mapping and interpolating by kriging. The loss of accuracy due to the interpolation by kriging is not an issue for most applications with parallel transmission [21].

The accuracy of the XFL sequence used in this paper can be improved: efforts are being made in this direction. If the experimental error can be reduced further and if better interpolation accuracy is desired, an anisotropic variogram could improve the kriging, but will be much more complicated to fit. Moreover, modeling the correlation structure between imaginary and real part or between channels could enhance the accuracy of the kriging algorithm, based on a multivariate variogram analysis and cokriging [29]. 
In vivo benchmarking was also attempted between standard XFL and SU-XFL sequences. The difference in channel-by-channel field maps also encompasses the error, difficult to estimate, due to displacements of the head between the two sequences. The displacements mainly explain the difference observed between XFL and SU-XFL. Moreover, it is well known that in vivo $B_{1}$ imaging is significantly less accurate than phantom $B_{1}$ imaging [18]. However, even under these circumstances, the $B_{1}$-calibration with the fast SU-XFL sequence appeared quite usable.

Even if the interpolation was proposed for the XFL-sequence, it can be directly applied to every mapping sequence using slice-selective pulses and 2-D-acquisitions. More generally, the kriging algorithm may help to shorten any MRI sequence, as long as the measured field varies slowly in space. For instance, it may be used for imaging $\mathrm{B}_{0}$ inhomogeneity [37], receive sensitivity [38], or even temperature [39]. The kriging technique can also be used for offline post-processing. This approach has already been demonstrated for component segmentation of rock samples [40]. Similarly, it could help for tissue segmentation in biomedical imaging.

To conclude, the slow variation in space of the field maps makes interpolation of low resolution field maps possible. Different approaches were proposed for interpolation and compared: kriging approach appeared the most accurate. We demonstrated its application to accelerate the XFL $B_{1}$-mapping sequence on phantom and volunteer. More generally, combining kriging technique to an accurate $B_{1}$-mapping sequence turns out to be a promising route to achieve fast and accurate $B_{1}$-calibration for multiple channel transmit array in high field MRI.

\section{ACKNOWLEDGMENT}

The authors would like to thank A. Lopez Kolkovsky for assisting with in vivo acquisitions on the 7T scanner.

\section{REFERENCES}

[1] J. Vaughan, M. Garwood, C. Collins, W. Liu, L. DelaBarre, G. Adriany, P. Andersen, H. Merkle, R. Goebel, M. Smith, and K. Ugurbil, "7 T vs $4 \mathrm{~T}$ : RF power, homogeneity and signal-to-noise comparison in head images," Magn. Reson. Med., vol. 46, no. 1, pp. 24-30, July 2001.

[2] D. Hoult and D. Phil, "Sensitivity and power deposition in a high field imaging experiment," J. Magn. Reson. Imaging, vol. 12, no. 1, pp. 46-67, July 2000.

[3] Y. G. A. Hyoungsuk and J. T. Vaughan, "A method to control non-uniformity RF B1 field for high field magnetic resonance imaging," in $\mathrm{Mi}$ crowave Symposium Digest (MTT), 2010 IEEE MTT-S International, Anaheim, CA, USA, 2010, pp. 752-755.

[4] A. Tannus and M. Garwood, "Adiabatic pulses," NMR in Biomedicine, vol. 10, no. 8, pp. 423-434, Dec. 1997.

[5] S. Saekho, C. Yip, D. C. Noll, F. E. Boada, and V. A. Stenger, "Fast-kz three-dimensional tailored radiofrequency pulse for reduced B1 Inhomogeneity," Magn. Reson. Med., vol. 55, no. 4, pp. 719-724, April 2006.

[6] N. Boulant, N. Mangin, and A. Amadon, "Counteracting RF inhomogeneity in the human brain at $7 \mathrm{~T}$ using strongly modulating pulses," Magn. Reson. Med., vol. 61, no. 5, pp. 1165-1172, May 2009.

[7] U. Katscher and P. Börnert, "Parallel RF transmission in MRI," NMR in Biomedicine, vol. 19, no. 3, pp. 393-400, May 2006.

[8] S.-M. Sohn, L. DelaBarre, J. T. Vaughan, and A. Gopinath, "RF multichannel head coil design with improved B1+ fields uniformity for high field MRI systems," in Microwave Symposium Digest (MTT), 2012 IEEE MTT-S International, Montreal, QC, Canada, 2012.
[9] W. Mao, M. B. Smith, and C. M. Collins, "Exploring the limits of RF shimming for high-field MRI of the human head," Magn. Reson. Med., vol. 56, no. 4, pp. 918-922, Oct. 2006.

[10] U. Katscher, P. Börnert, C. Leussler, and J. S. Van den Brink, "Transmit SENSE," Magn. Reson. Med., vol. 49, no. 1, pp. 144-150, Jan. 2003.

[11] Y. Zhu, "Parallel excitation with an array of transmit coils," Magn. Reson. Med., vol. 51, no. 4, pp. 775-784, April 2004.

[12] K. Setsompop, V. Alagappan, B. Gagoski, T. Witzel, J. Polimeni, A. Potthast, F. Hebrank, U. Fontius, F. Schmitt, L. L. Wald, and E. Adalsteinsson, "SlicesSelective RF pulses for in vivo B1+ inhomogeneity mitigation at 7 Tesla using parallel RF excitation with a 16-element coil," Magn. Reson. Med., vol. 60, no. 6, pp. 1422-1432, Dec. 2008.

[13] K. Setsompop, L. L. Wald, V. Alagappan, B. A. Gagosku, and E. Adalsteinsson, "Magnitude least squares optimization for parallel RF excitation design demonstrated at 7 T with 8 channels," Magn. Reson. Med., vol. 59, no. 4, pp. 908-915, April 2008.

[14] F. Hennel, "Method for Mapping of the Radio Frequency Field Amplitude in a Magnetic Resonance Imaging System Using Adiabatic Excitation Pulses," US Patent US20100237861 A1, Sep. 23, 2010.

[15] J.-Y. Park and M. Garwood, "B1 mapping using phase information created by frequency-modulated pulses," in Proc. Intl. Soc. Mag. Reson. Med., Toronto, Canada, 2008, p. 361.

[16] L. I. Sacolick, F. Wiesinger, I. Hancu, and M. W. Vogel, "B1 mapping by bloch-Siegert shift," Magn. Reson. Med., vol. 63, no. 5, pp. 1315-1322, May 2010.

[17] V. Yarnykh, "Actual flip-angle imaging in the pulsed steady state," Magn. Reson. Med., vol. 57, no. 1, pp. 192-200, Jan. 2007.

[18] S. Chung, D. Kim, E. Breton, and L. Axel, "Rapid B1+ mapping using a preconditioning RF pulse with TurboFLASH readout," Magn. Reson. Med., vol. 64, no. 2, pp. 439-446, Aug. 2010.

[19] H. P. Fautz, M. Vogel, P. Gross, A. Kerr, and Y. Zhu, "B1 mapping of coil arrays for parallel transmission," in Proc. Intl. Soc. Magn. Reson. Med., Toronto, Canada, 2008, p. 1247.

[20] A. Amadon, N. Boulant, M. A. Cloos, E. Giacomini, C. J. Wiggins, M. Luong, G. Ferrand, and H. P. Fautz, "B1-mapping of an 8-channel TX-array over a human-head-like volume in less than 2 minutes: The XEP sequence," in Proc. Intl. Soc. Mag. Reson. Med., Stockholm, Sweden, 2010, p. 18.

[21] A. Amadon, M. A. Cloos, N. Boulant, M. Hang, C. J. Wiggins, and H. P. Fautz, "Validation of a very fast B1-mapping sequence for parallel transmission on a human brain at $7 \mathrm{~T}$," in Proc. Intl. Soc. Magn. Reson. Med., Melbourne, Australia, 2012, p. 4885.

[22] D. A. Feinberg, A. Beckett, and L. Chen, "Arterial spin labeling with simultaneous multi-slice echo planar imaging," Magn. Reson. Med., vol. 70, no. 6, pp. 1500-1506, Dec. 2013.

[23] C. H. Cunningham, J. M. Pauly, and K. S. Nayak, "Saturated doubleangle method for rapid B1+ mapping," Magn. Reson. Med., vol. 55, no. 6, pp. 1326-1333, June 2006.

[24] A. Sbrizzi, H. Hoogduin, J. J. Lagendijk, P. Luitjen, and C. A. T. Van den Berg, "Robust reconstruction of B1+ maps by projection into a spherical functions space," Magn. Reson. Med., vol. 71, pp. 394-401, Jan. 2014.

[25] G. Matheron, "Principles of geostatistics," Economic Geology, vol. 58, pp. 1246-1266, 1963.

[26] O. Baume, B. Gauvreau, M. Bérengier, F. Junker, H. Wackernagel, and J. P. Chilès, "Geostatistical modeling of sound propagation: Principles and a field application experiment," Acoustical Society of America, vol. 126, pp. 2894-2904, 2009.

[27] J. Ruiz-Alzola, C. F. Westin, S. K. Warfield, C. Alberola, S. Maier, and R. Kikinis, "Non-rigid registration of 3-D tensor medical data," Medical Image Analysis, vol. 6, pp. 143-161, 2002.

[28] J. Lefëbvre, H. Roussel, E. Walter, D. Lecointe, and W. Tabbara, "Prediction from wrong models: The Kriging approach," IEEE Antennas Propagat. Mag., vol. 38, no. 4, pp. 35-45, Aug. 1996.

[29] H. Wackernagel, Multivariate Geostatistics. : Springer Verlag, 2003.

[30] P. Thévenaz, T. Blu, and M. Unser, "Interpolation revisited," IEEE Trans. Med. Imag., vol. 19, no. 7, pp. 739-758, July 2000.

[31] L. P. Yaroslavsky, "Efficient algorithm for discrete sinc interpolation," Applied Optics, vol. 36, no. 2, pp. 460-463, Jan. 1997.

[32] K. Nehrke and P. Börnert, "Eigenmode analysis of transmit coil array for tailored B1 mapping," Magn. Reson. Med., vol. 63, no. 3, pp. 754-764, March 2010.

[33] D. Brunner and K. Pruessmann, "B1 interferometry for the calibration of RF transmitter arrays," Magn. Reson. Med., vol. 61, no. 6, pp. 1480-1488, June 2009. 
[34] M. A. Cloos, N. Boulant, M. Luong, G. Ferrand, E. Giacomini, D. Le Bihan, and A. Amadon, "kt-points: Short three-dimensional tailored RF pulses for flip-angle homogenization over an extended volume," Magn. Reson. Med., vol. 67, no. 1, pp. 72-80, Jan. 2012.

[35] M. Zhadobov, G. Ferrand, M. Luong, Y. Soubere-Mahamoud, C. C. P Le Quement, Y. Piret, R. Sauleau, and Y. Le Drean, "Exposure system and dosimetry for in vitro studies of biocompatibility of pulse-modulated RF signals of ultra high field MRI," IEEE Trans. Biomed. Eng., vol. 60 , no. 11, pp. 3167-3175, Nov. 2013.

[36] G. Ferrand, M. Luong, A. C. M. A. Amadon, E. Giacomini, and L. Darrasse, "Generalized double-acquisition imaging for radiofrequency inhomogeneity mitigation in high-field MRI: Experimental proof and performance analysis," Magn. Reson. Med., vol. 67, no. 1, pp. 175-182, Jan. 2012

[37] M. Schär, E.-J. Vonken, and M. Stuber, "Simultaneous B0- and B1+-map acquisition for fast localized shim, frequency, RF power determination in the heart at 3 T," Magn. Reson. Med., vol. 63, no. 2, pp. 419-426, Feb. 2010.
[38] J. Wang, M. Qiu, Q. X. Yang, M. B. Smith, and R. T. Constable, "Measurement and correction of transmitter and receiver induced non-uniformities in vivo," Magn. Reson. Med., vol. 53, no. 2, pp. 408-417, Feb. 2005.

[39] S. Hey, M. de Smet, C. Stehning, H. Grüll, J. Keupp, C. Moonen, and M. Ries, "Simultaneous T1 measurements and proton resonance frequency shift based thermometry using variable flip angles," Magn. Reson. Med., vol. 67, no. 2, pp. 457-463, Feb. 2012.

[40] W. Oh and W. B. Lindquist, "Image thresholding by indicator Kriging," IEEE Trans. Pattern Anal. Machine Intell., vol. 21, no. 7, pp. 590-602, July 1999. 